\title{
Two-parameter sequential adsorption model applied to microfiber clustering
}

\section{Citation}

Paulose, Jayson, David R. Nelson, and Joanna Aizenberg. 2010. “Two-Parameter Sequential Adsorption Model Applied to Microfiber Clustering." Soft Matter 6 (11): 2421. doi:10.1039/ c000443j.

\section{Published Version}

doi:10.1039/C000443J

\section{Permanent link}

http://nrs.harvard.edu/urn-3:HUL.InstRepos:37256295

\section{Terms of Use}

This article was downloaded from Harvard University's DASH repository, and is made available under the terms and conditions applicable to Open Access Policy Articles, as set forth at http:// nrs.harvard.edu/urn-3:HUL.InstRepos:dash.current.terms-of-use\#OAP

\section{Share Your Story}

The Harvard community has made this article openly available.

Please share how this access benefits you. Submit a story.

Accessibility 


\title{
Two-parameter sequential adsorption model applied to microfiber clustering
}

\author{
Jayson Paulose, ${ }^{* a}$ David R. Nelson, ${ }^{a b}$ and Joanna Aizenberg ${ }^{a c}$
}

\author{
Received Xth $X X X X X X X X X X 20 X X$, Accepted Xth $X X X X X X X X X 20 X X$ \\ First published on the web Xth $X X X X X X X X X X 200 X$ \\ DOI: 10.1039/b000000x
}

Capillary-mediated self-assembly and self-organization are useful techniques for constructing ordered superstructures from nanoscale and microscale building blocks. Square arrays of flexible microfibers attached to a substrate have been shown to form highly ordered patterns of 2x2 fiber clusters (tetramers) under the influence of capillary forces at the surface of an evaporating liquid layer. We model this pattern formation as an irreversible sequential adsorption process on a square lattice, in which tetramers form sequentially on an initially empty lattice and locally enhance the formation of nearby tetramers, giving rise to partially ordered domains. Restrictions analogous to excluded volume interactions for hard squares prevent additional tetramers forming at next-and second-neighbor positions. Two parameters regulate the enhancement in tetramer formation at third- and fourth-near neighbor positions. We study the model using numerical simulations and compare it to a realization of a self-organization experiment. The model reproduces many features of the observed patterns when the two parameters are chosen by a least-squares fit to a single experimental quantity. The fourth-near neighbor enhancement, not considered in previously studied sequential adsorption models, is shown to be significant for the pattern formation under study.

\section{Introduction}

The study of the self-assembly and self-organization of solid macro-, micro- and nanoscale structures using capillary forces mediated by a wetting liquid has made an impact in fields ranging from photonics ${ }^{1}$ to the theory of computation. ${ }^{2}$ In recent years, the phenomenon of elastocapillary coalescence ${ }^{3}$ has been observed in aggregation of fibers ranging in size from the macroscopic scale ${ }^{4,5}$ down to micro- and nanoscales. ${ }^{6-13}$ In all these investigations, capillary forces in a wetting liquid bring together the free ends of high-aspect-ratio structures attached to a substrate to form clusters. For highly symmetrical arrangement of fibers in the individual clusters, a long-range ordering in the positions of the clusters has been reported. ${ }^{10-13}$ In particular, when the fibers are initially arranged in a square lattice and primarily form $2 \times 2$ clusters of four individual fibers connected at the tip, these $2 \times 2$ clusters themselves are ordered locally in a square superlattice with a lattice spacing twice that of the individual fibers (figure 1(a)). This ordering mechanism has potential application not only as a means of creating complex three-dimensional structures with spatial regularity but also as a basis for generating dynamic "smart" surfaces capable of ordered particle trapping and release, color changes and

a School of Engineering and Applied Sciences, Harvard University, Cambridge, MA 02138, USA. E-mail: jpaulose@fas.harvard.edu

${ }^{b}$ Department of Physics, Harvard University, Cambridge, MA 02138, USA.

${ }^{c}$ Department of Chemistry and Chemical Biology, Harvard University, Cambridge, MA 02138, USA. adaptive wetting behavior. ${ }^{12,14}$

A number of previous theoretical treatments of capillaryassisted clustering ${ }^{5,6,8-10,15}$ have analyzed the elastic, capillary and surface tension energies associated with fiber clustering to determine the critical stiffness of the fibers that allows clustering to occur, and the typical size/size distribution of clusters. However, less has been said about the higher-level arrangement, size and ordering of the clustered domains. A qualitative picture of long-range ordering of clusters due to the distance dependence of the capillary interactions between submerged fibers is presented in Ref. 12, and a similar description based on the stability of upright fibers is reported in Ref. 13, but to our knowledge no quantitative comparison of these descriptions with experimentally observed patterns has been made. The current work develops this aspect of capillarymediated clustering of fibers. We propose, characterize and test a nonequilibrium dynamical model for the irreversible formation of clusters showing long-range order in a square array of fibers.

Our approach falls into a broad class of systems known as sequential adsorption models. ${ }^{16}$ Sequential adsorption (SA) models describe deposition of particles on a surface through the following algorithm: a trial deposition of a particle is made on the surface (typically empty initially, though a 'seeded' surface with predeposited particles may also be studied); if the particle does not overlap with any other, the deposition is successful and the particle is permanently fixed in the chosen spot; if on the other hand the particle overlaps with an already 


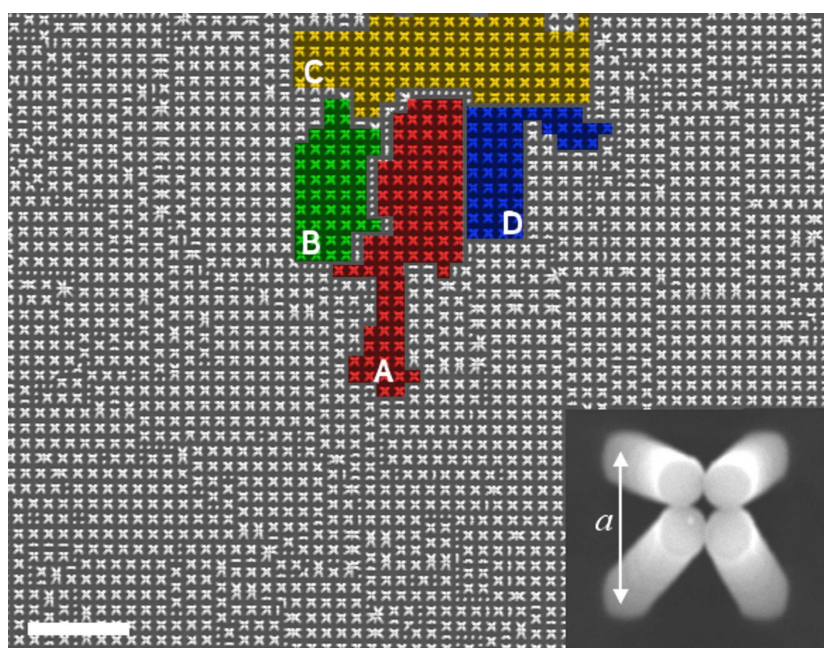

(a)

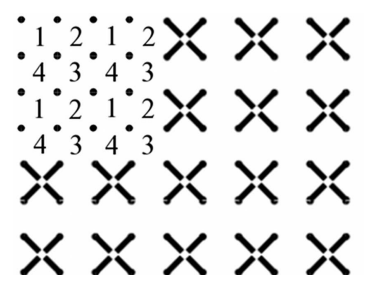

(b)

Fig. 1 (a) Top-down scanning electron microscope (SEM) image of a region of an epoxy microfiber sample in which clusters have been induced via ethanol evaporation and persist due to van der Waals interactions between the touching fiber tips. Scale bar $50 \mu \mathrm{m}$. The region predominantly shows tetramers, i.e. clusters of four fibers whose tips meet at a height of a few microns above the substrate, centered above the squares of the underlying lattice of fibers. The inset (bottom right) is a close-up view of a tetramer, showing the lattice spacing $a$ of the fibers. Four distinct domains (as defined in the text) are highlighted and labeled A-D. (b) Schematic of the lattice of fibers with some formed tetramers all belonging to a single domain. (In this and following diagrams, each dot represents the base of an upright fiber while each ' $\mathrm{x}$ ' represents the top-down view of a tetramer comprising of four fibers whose bases remain on the square lattice but tips come together at the center.) The numbers show the division of the dual lattice into four sublattices 1-4 corresponding to the four distinct domains shown in (a); the remainder of the lattice is occupied by a domain whose members occupy sublattice 1 . In the SEM image, we may assign the tetramers of domain A to sublattice 1; domains $\mathrm{B}, \mathrm{C}$ and $\mathrm{D}$ then belong to sublattices 2, 3 and 4 respectively.

attached particle then the trial is rejected. Random sequential adsorption (RSA) models refer to those in which the position of the trial deposition is chosen purely at random, while cooperative sequential adsorption (CSA) processes are those in which the probability of a trial deposition at any point on the surface is influenced (typically enhanced) by the presence of deposited particles nearby. Both RSA and CSA, on continuum surfaces as well as lattices, have been used to study a variety of physical, chemical and biological processes (for a comprehensive review see Ref. 16). The questions of interest in studying such models range from predicting the final coverage of the surface when the system has evolved until no further deposition takes place (i.e. there is no further room for particle deposition and the system reaches a 'jammed' or 'saturated' state) to understanding the time evolution up to jamming as well as the spatial distributions and correlations of the particles at any stage.

Since the deposition events are irreversible, sequential adsorption models are typically not amenable to study via tools of equilibrium statistical mechanics. ${ }^{16}$ Theoretical modeling begins with the formulation of rate equations describing the filling of empty areas of the surface with particles. The result is a hierarchical set of rate equations linking each empty configuration to the set of empty configurations that could result from the adsorption of a particle anywhere within it. These equations describe a nonequilibrium generalization of the famous dimer problem of statistical physics, ${ }^{17}$ with dimers being replaced by hard spheres in our case. One-dimensional RSA models as well as CSA models with short-range cooperative effects are exactly solvable. However, in dimensions two and higher, the hierarchy of rate equations becomes very large and is not exactly solvable even for the simplest short-range cooperative effects (such as nearest-neighbor exclusion on a lattice). A variety of approximation methods such as series expansions for small coverages (i.e. early times) and systematic truncation of the hierarchical rate equations has been adopted to study higher dimensional RSA/CSA models. The algorithmic nature of the SA process also makes it a good candidate for direct numerical simulation on finite-sized lattices or surfaces, which is the approach taken by this paper.

With experimental patterns like the one depicted in figure 1(a) in mind, we develop here a model of irreversible CSA of particles on an initially empty square lattice, under the conditions that adsorption of a particle at any site prevents further adsorption not only at the filled site but at nearest neighbor $(\mathrm{NN})$ and next-nearest neighbor $(2 \mathrm{NN})$ positions, but increases the rate of adsorption at the third (3NN) and fourth (4NN) nearest neighbor positions. (As explained in detail in section 2.1, these lattice positions correspond to the positions of plaquettes of the microfiber array for the particular case of fiber clustering; representative NN through 4NN positions relative to a $2 \times 2$ cluster are indicated in figure 2(b).) Local cooperativity in the adsorption process is typically introduced by defining adsorption rates that depend on the local environment of each site. ${ }^{16}$ For our model, we assign a rate $k(i, j)=k_{0} \alpha^{i} \beta^{j}$ to each site that depends on the numbers $i$ and $j$ of particles previously adsorbed at $3 \mathrm{NN}$ and $4 \mathrm{NN}$ positions respectively 
relative to the site. Enhancement of adsorption near previously adsorbed particles corresponds to $k(i, j)>k(0,0)$ for allowed values of $(i, j)$ other than $(0,0)$. Here $k(0,0) \equiv k_{0}$ is the rate of adsorption of particles for a site that has no filled $3 \mathrm{NN}$ or $4 \mathrm{NN}$ sites. We shall take $\alpha$ and $\beta$ greater than 1 , so that they describe in a simple way enhanced "adsorption" (tetramer clustering in our case) at $3 \mathrm{NN}$ and $4 \mathrm{NN}$ positions respectively. While the lone particle adsorption rate $k_{0}$ determines the overall speed at which the surface is covered with particles, the nature of the adsorbed pattern at any fractional coverage and particularly at saturation depends only on $\alpha$ and $\beta$ which are the two adjustable parameters in the model.

Our model is similar to previously studied CSA models on a square lattice with close-neighbor cooperative effects. ${ }^{16,18-21}$ Previous studies, however, have focused on a single level of cooperativity with rates $k_{i}=\alpha^{i} k_{0}, \alpha>1$ defined at each site when $i$ is the number of occupied closest neighbor sites. (This rate choice is called a multiplicative or Arrhenius rate, in contrast to the Eden rate choice, $k_{i}=\alpha k_{0}, \alpha>1$ for $i \geq 1$.) Some of these analyses are also useful in explaining the properties of our model. For example, in the regime of strong cooperativity when adsorption near occupied sites is strongly favored, adsorbed particles tend to form growing domains or islands 19,20 around a randomly deposited nucleation site. Here we define a domain as a group of tetramers whose centers may be connected to each other by a network of bonds each of length $2 a$, where $a$ is the lattice spacing of the square array of fibers. As shown in figure 1, the domains lie on one of four sublattices and domains belonging to different sublattices meet in boundaries that largely follow the principal axes of the lattice of fibers. Then $k_{0}$ may be considered the rate of nucleation of domains while the other rates describe the growth of domains. As adsorption continues, growing domains on the same sublattice coalesce upon meeting one another, while domains belonging to different sublattices meet at a frozen domain boundary. As long as all $k(i, j)>0$, the system ultimately reaches a 'saturated' or 'jammed' state at which point no further adsorption can take place and the state of the entire lattice may be described by domain boundaries separating domains of various shapes and sizes. This saturated state is the one that is compared to the experimental domain patterns. In the fiber clustering experiments discussed above, rare anomalous clusters of $2,3,5,6, \ldots$ fibers constitute additional point-like defects which we disregard here.

In section 2, we summarize the experiments leading to the self-organization of microfibers and motivate the CSA model with NN/2NN exclusion and 3NN/4NN cooperative effects to describe ordering and domain formation in this system. In section 3, we characterize the patterns of tetramers generated by the model for a range of parameter values using computer simulations. We emphasize the effects of the newly considered $4 \mathrm{NN}$ cooperativity $(\beta>1)$ to contrast the current model with previously studied single-parameter models, and highlight the similarity of the tetramer-tetramer correlation functions for different values of $\alpha$ and $\beta$ upon appropriate rescaling. In section 4 , we evaluate the ability of the model to reproduce patterns from an actual experiment, using a single measured quantity to fit the two parameters $\alpha$ and $\beta$, and compare the model's performance to that of a similar single-parameter model. We show that a single-parameter model deviates significantly from the experimental observations, while our new double-parameter model provides an excellent description of the experimentally observed patterns. In section 5 , we conclude by using the insights provided by the model to improve ordering in the clustering experiment, and consider the potential relevance of the new model to various sequential adsorption processes and self-assembly. Details of the numerical simulation and the experimental procedure are reported in appendices $\mathrm{A}$ and $\mathrm{B}$ respectively.

\section{Microfiber clustering and order formation}

The formation of large ordered regions during selforganization of nanostructures via capillary forces has been reported by the Aizenberg group in Ref. 12. In this section we summarize the experiment and observations that motivate our theory. Highly uniform square arrays of high-aspect-ratio nano- and microscale polymeric fibers, prepared using soft lithography as described in Ref. 22, were wet with a solvent which was allowed to evaporate under ambient conditions. As the solvent-air interface is forced below the tips of the upright fibers due to evaporation, the tips are drawn together by capillary interactions and adhere to each other upon contact via short-range van der Waals forces, forming clusters that persist after all the liquid has evaporated and may be observed by optical and scanning electron microscopy. Under certain conditions of fiber geometry and stiffness, the clusters formed could be primarily tetramers composed of four fibers meeting at the tips. The tetramers were arranged in highly regular arrays, with large domains (spanning several lattice lengths) composed of groups of tetramers ordered in a square superlattice of lattice constant twice the distance between fibers. A representative SEM image of such a system after clustering is shown in figure 1(a).

\subsection{Ordering mechanism and formulation of the CSA model}

A qualitative one-dimensional mechanism for the formation of ordered domains has been proposed ${ }^{12}$ that propagates a breaking of the lattice symmetry into one sublattice due to the nature of the attractive capillary forces between fiber tips. For small displacements of the meniscus from the horizontal plane, the capillary forces are proportional to $\gamma r^{2} / d$, where 


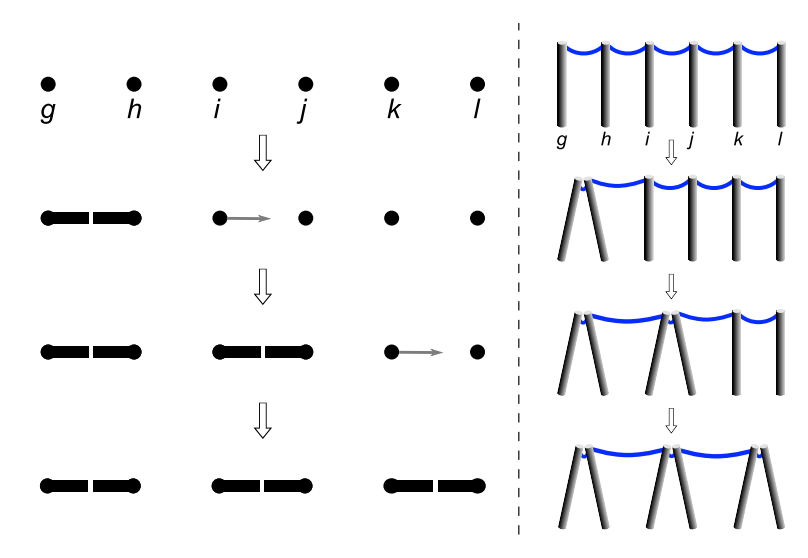

(a)

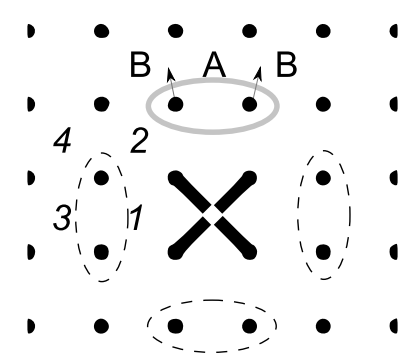

(b)

Fig. 2 Capillary-mediated cooperative clustering. (a) illustrates the mechanism in a one-dimensional row of fibers whose tips are drawn together to form dimers (top view, left; side view, right). The large dots in the top view indicate the bases of upright fibers. When the liquid-air interface first reaches the upright fiber tips, all interfiber forces are equal. Now suppose the random formation of a cluster brings fibers $g$ and $h$ together. The tips of $i$ and $h$ are now further apart than the tips of $i$ and $j$; hence $i$ experiences an imbalance of forces (grey arrow) that induces it to cluster with its neighbor $j$. Now fiber $k$ experiences a force imbalance, and the effect of the initial clustering of $g$ and $h$ thus cascades down the lattice. (b) extends the picture to tetramer formation in two dimensions (top view). Representative first through fourth neighbor squares or "plaquettes" relative to the nucleating tetramer are indicated by numbers 1-4. Due to the formation of a tetramer, the four highlighted pairs of fibers experience a force imbalance. As shown for the top pair, there is a resultant force on each fiber due to capillary attraction with the nearby fibers and tetramer (whose fibers perturb the ethanol-air interface further away compared to the unclustered fibers). This increases the probability of tetramer formation at the 'A' position (third-nearest neighbor), and to a smaller extent at the ' $\mathrm{B}$ ' positions (fourth-nearest neighbors).

$\gamma$ is the surface tension of the ethanol-air interface, $r$ the radius of the fibers and $d$ the distance between fiber tips at the meniscus. ${ }^{23}$ The cooperativity in cluster formation arises as a result of the inverse relationship between interfiber distance $d$ and capillary force between fiber tips. Figure 2(a) illustrates this mechanism. If a dimer nucleates due to an imperfection or instability (such as a nonuniformity in the rate of evaporation of the ethanol, or slight variations in the spacing of the fiber tips when they encounter the ethanol-air interface), that event induces a fiber to buckle or bend, and the fiber to the right of the dimer then experiences a net force towards its own neighbor on its right which is closer to it than the fiber participating in the dimer on its left. Thus, the initial dimerization induces the fiber to form a new dimer with the fiber on its right. This cascade then continues, propagating a chain of dimers with periodicity $2 a$. To extend this picture to two dimensions, note that the formation of a tetramer has the biggest effect on four pairs of neighboring fibers in the four cardinal directions. For instance, consider the highlighted pair of fibers in figure 2(b). Due to the force imbalance induced by the initial tetramer, the pair moves toward the fibers to its north, increasing the probability of forming a tetramer in position ' $\mathrm{A}$ ' which is a third-nearest-neighbor, or $3 \mathrm{NN}$, position relative to the formed tetramer. However, each destabilized fiber also has an enhanced probability of forming a tetramers at the two positions marked 'B', the $4 \mathrm{NN}$ positions relative to the formed tetramer. Hence the effect of the formed tetramer on the fibers closest to it is fully captured if we include both $3 \mathrm{NN}$ and $4 \mathrm{NN}$ enhancement in tetramer formation. We would expect the effect to be weaker for $4 \mathrm{NN}$ tetramers, but to increase when the number of $3 \mathrm{NN}$ or $4 \mathrm{NN}$ neighbors is increased as more fibers are perturbed by force imbalances. (Note that tetramers cannot form at $\mathrm{NN}$ or $2 \mathrm{NN}$ positions due to a lack of a complete set of four upright fibers. Also, the absence in the experimental patterns of adjacent pairs of dimers suggests that any dimer formed near an initial tetramer immediately forms a more stable tetramer configuration with the next pair of fibers if it is available, giving rise to a $3 \mathrm{NN}$ or $4 \mathrm{NN}$ cluster. Hence we do not include the possibility of dimer formation induced by a tetramer in neighboring fibers.)

Thus the formation of ordered domains occurs in the following way: random instabilities nucleate lone tetramers in the lattice of initially upright fibers at some background rate. Once a tetramer is formed, the nature of capillary interactions enhances the rate at which tetramers are formed at $3 \mathrm{NN}$ and $4 \mathrm{NN}$ positions near it. The $3 \mathrm{NN}$ enhancement tends to be stronger, giving rise to the formation of ordered domains of tetramers that grow from different nucleating sites. Nucleation of a $4 \mathrm{NN}$ tetramer at the edge of a growing domain disrupts its growth, instead starting a domain belonging to a different sublattice. Once tetramers are formed, they do not unbind.

Note that formation of tetramers on the square lattice of fibers is equivalent to the deposition of single particles on sites of the dual square lattice (which is the lattice formed from the centers of the plaquettes formed by the fiber lattice, or equivalently the fiber lattice displaced by $a / 2$ in both the $x$ and $y$ directions). Hence the irreversible process of tetramer formation 
as described above is equivalent to a cooperative sequential adsorption process of particles that occupy sites on a square lattice with $\mathrm{NN} / 2 \mathrm{NN}$ exclusion and $3 \mathrm{NN} / 4 \mathrm{NN}$ enhancement of deposition. The initial state of upright fibers (no tetramers) is equivalent to an empty initial lattice for particle deposition. The rate of deposition at each site at any instant in time is determined by the previously deposited particles (if any) at 3NN and $4 \mathrm{NN}$ positions, or is equal to the rate of random or bare nucleation if there are no such deposited neighbors. The final state after all the ethanol has evaporated, when all fibers are clustered except those that do not have enough neighbors to form tetramers, corresponds to a state of jamming or saturation for adsorption on the dual lattice.

\subsection{Choice of CSA model rates}

To complete our model, we must specify the localenvironment-dependent rate of adsorption of tetramers at empty sites. The transformation of a group of four upright fibers to a tetramer happens in less than a hundredth of a second, ${ }^{24}$ practically instantaneous over the time scale of the clustering of the entire sample (roughly a minute). We assume that the formation of a tetramer at a particular site on the (dual) lattice is a stochastic process with a waiting time that is exponentially distributed with a mean time $\tau$, which depends on the geometry of the fibers, the surface and bulk properties of the fiber material, the surface tension of the evaporating liquid and the level of the ethanol-air interface, as well as the configuration of fibers and/or tetramers at nearby positions. The rate of formation (adsorption) at the particular site is then $1 / \tau$. We do not attempt to calculate these rates microscopically for different local environments but treat them as parameters that we extract from a particular experimental realization by fitting to simulation results.

Even if we restrict ourselves to $3 \mathrm{NN} / 4 \mathrm{NN}$ effects of previously formed tetramers, there are several different rates of adsorption to an empty site based on different possible configurations of previously adsorbed tetramers around it. For instance, there are five different configurations involving one, two, three or four tetramers adsorbed at $3 \mathrm{NN}$ sites that are not superimposable via rotations, and several more independent configurations when considering all possible combinations of adsorbed 3NN/4NN tetramers allowed under the exclusion rule. To reduce the number of independent parameters in the model, we make the simplifying assumption that the adsorption rate $k(i, j)$ at any site is a function only of the number of tetramers adsorbed at $3 \mathrm{NN}$ and $4 \mathrm{NN}$ positions ( $i$ and $j$ respectively) and not their spatial arrangement around the site.

The functional dependence of the rate on $i$ and $j$ (which, of course, take only non-negative integer values) is motivated by the observation of long unbroken domain walls along both lattice directions, which suggests that individual domains grow- ing from different nucleation sites are largely rectangular in shape when they encounter one another. Let us focus initially on the stronger $3 \mathrm{NN}$ cooperativity, ignoring any dependence of the rates on $j$ so that $k(i, j) \equiv k_{i}$. Then $k_{0}, k_{1}$ and $k_{2}$ represent respectively the rates of nucleation of a new domain, beginning of a new row at the edge of a growing domain, and growth of an incomplete row at the edge of a domain by formation of a tetramer at either end of the row. The ratio $k_{1} / k_{0}$ determines the average size to which domains grow before they encounter other domains and stop, while $k_{2} / k_{1}$ determines the size until which a growing domain remains rectangular. ${ }^{*}$ Evans and Nord ${ }^{21}$ have shown that multiplicative rates that satisfy $k_{0}: k_{1}: k_{2}=1: \alpha: \alpha^{2}$ with $\alpha>1$ give rise to domains that maintain their rectangular shape until saturation is reached in the adsorption process. In contrast, a weaker arithmetic increase in cooperativity with $i$ such as $k_{0}: k_{1}: k_{2}=1: \alpha: 2 \alpha$ would lead to patterns with irregular domain walls as domains do not maintain their rectangular shape. Motivated by these observations and the experiments themselves, we choose multiplicative rates that independently grow with $i$ and $j: k(i, j)=k_{0} \alpha^{i} \beta^{j}$, where $\alpha$ and $\beta$ are the cooperativity parameters associated with $3 \mathrm{NN}$ and $4 \mathrm{NN}$ tetramers respectively. This simplified form has several advantages: it reduces the number of parameters to two while retaining the desired properties of two cooperativity levels, cooperativity that increases with number of adsorbed neighbors, and support for rectangular domains; the levels of $3 \mathrm{NN}$ and 4NN cooperativity may be independently varied; and the similarity with (one-parameter) multiplicative rates allows a comparison with models that have been previously studied. We do not propose that the actual microscopic rates in experiment follow this choice of rates, but rather that this simplified choice reflects the local environment dependence of the actual rates sufficiently well to recreate key features of the patterns seen in experiment - particularly the relative influence of $3 \mathrm{NN}$ and 4NN cooperativity. As we shall see, a large number of experimental features can be understood upon adjusting just these two parameters.

\section{Simulation results}

We study the proposed CSA model using Monte Carlo simulations, described in brief in Appendix A. The simulations were carried out on $400 \times 400$ lattices, with results averaged across 100 runs for each $(\alpha, \beta)$ combination.

\footnotetext{
* The time taken to complete an edge on a rectangular domain with edge length $m$ is $t_{c} \sim m / k_{2}$; in that time, the rectangular shape would be spoiled if a new edge is begun before the whole string of $m$ tetramers is added to complete the growing edge. This happens $\sim m k_{1} t_{c} \approx m^{2} k_{1} / k_{2}$ times; hence the rectangular shape of the domain can be maintained only for $m^{2} k_{1} / k_{2} \lesssim 1$ i.e. $m \lesssim \sqrt{k_{2} / k_{1}}$. Discussed in Ref. 21.
} 

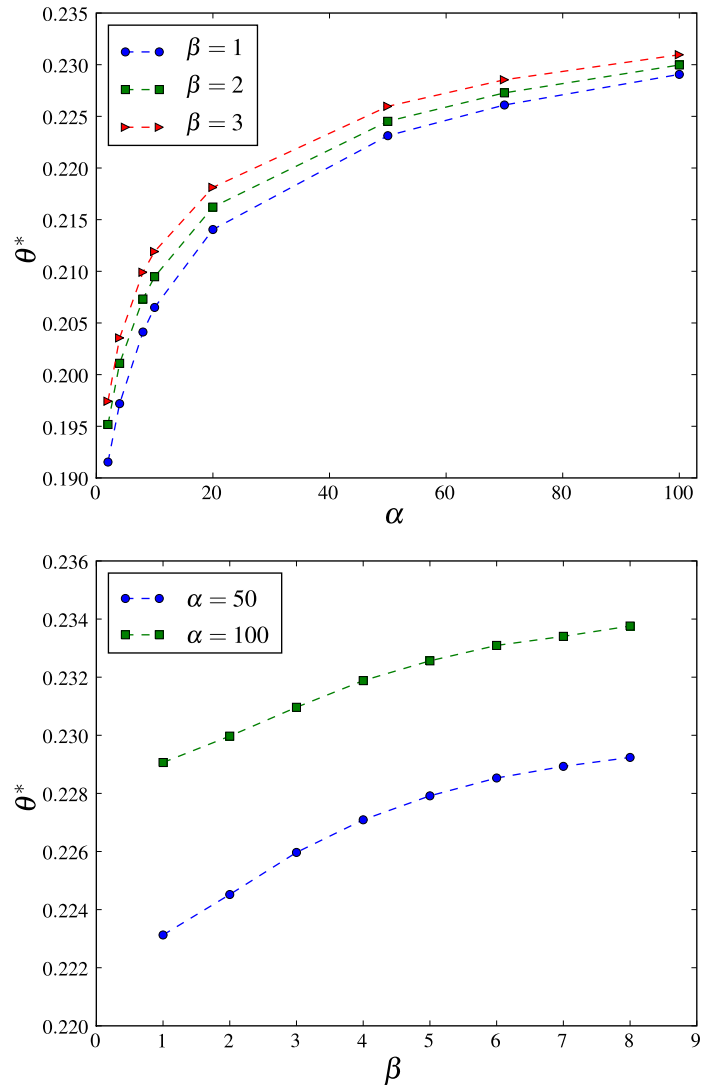

Fig. 3 Dependence of saturation coverage, $\theta^{*}$, on cooperativity parameters $\alpha$ and $\beta$ in the CSA model (simulation). The top figure highlights the $\alpha$-dependence when $\beta$ is kept constant and vice-versa on the bottom. The lines are guides to the eye.

\subsection{Mean coverage at saturation}

The coverage $\theta$ at any point during the adsorption process is the fraction of filled sites. The saturation coverage $\theta^{*}$ is the coverage when no further particles can adsorb. The maximum possible coverage is achieved when the entire lattice consists of one single domain, in which case $\theta^{*}=1 / 4$ (the NN and $2 \mathrm{NN}$ exclusion allows at most a fourth of the dual lattice to be covered in tetramers, all on one sublattice).

The saturation coverage is expected to increase with the cooperativity, because deviations from the maximum possible value occur only when domains with different nucleating sites coalesce. A higher rate of tetramer formation near previously formed tetramers (compared to nucleation of lone tetramers) ensures that the dual lattice is filled with fewer domain walls; i.e. higher values of $\alpha$ and $\beta$ give rise to higher values of saturation coverage, as seen in figure 3 .

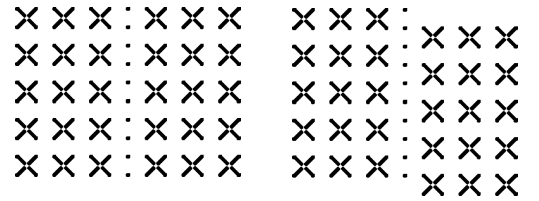

(a) (b)

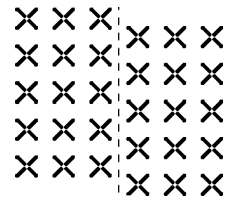

(c)
Fig. 4 Domain walls between tetramer domains. (a) and (b) depict Type I domain walls that leave behind upright fibers (depicted by dots) which are not part of any cluster. On the dual lattice, two empty vertical rows separate the domains. The domain on the left in (a) belongs to sublattice 1 (if numbering begins from the top left as in figure 1(b)) while the domain on the right belongs to sublattice 2 . In (b), the domain boundary is between domains on sublattice 1 and sublattice 3. (c) depicts a vertical Type II domain wall (dashed line) that does not leave behind any unclustered fibers. The domains are separated by only one row of empty sites on the dual lattice. The boundary is between a domain on sublattice 1 and one on sublattice 4. There are, of course, horizontal analogs of all three types of wall.

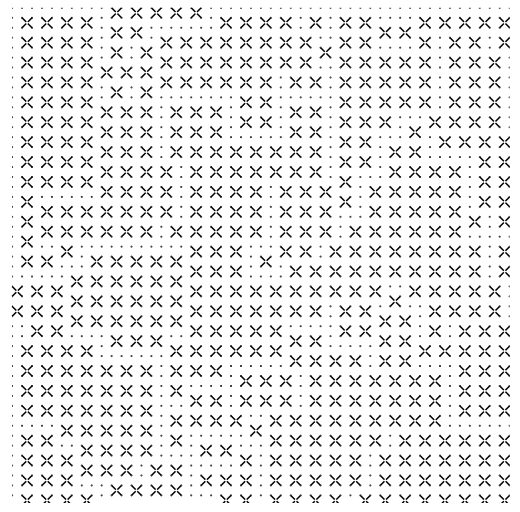

Fig. 5 A typical result of the CSA process run to saturation has a mixture of Type I and Type II domains in both the horizontal and vertical directions. "Zig-zag" walls are occasionally seen which may be considered a combination of horizontal and vertical sections.

\subsection{Domain sizes, chord lengths and domain wall densi- ties}

We have already defined domains as groups of particles (i.e. tetramers) connected to each other by a network of $3 \mathrm{NN}$ bonds. Domains belonging to different sublattices meet in domain walls that also run along the principal lattice directions. As figure 4(a-c) shows, there are two types of boundaries between domains that occur at saturation: boundaries involving free-standing single fibers that do not have enough neighbors to form tetramers (Type I) and boundaries that do not have such freely standing fibers (Type II). As a result of the CSA process, patterns with several domains meeting in boundaries of either type that primarily follow the principal directions of 
the lattice are generated (figure 5). "Zig-zag" domain walls that follow diagonal directions in the lattice are rare because of the Arrhenius choice of rates. ${ }^{\dagger}$

Once the different domains in the simulation result and the domain boundaries between them have been identified, ensemble-averaged measures of the domain size and the domain boundary lengths may be obtained. For a given simulation run, if $n_{s}$ is the number of domains with $s$ particles in them, we define the average domain size $s_{\mathrm{av}}=\sum s n_{s} / \sum n_{s}$. We also consider the statistics of adsorbed particles on a row-byrow or column-by-column basis. Defining a chord as a contiguous string of particles connected by $3 \mathrm{NN}$ bonds in the vertical or horizontal direction of the lattice, we may also measure the average chord length $m_{\mathrm{av}}=\sum m n_{m} / \sum n_{m}$ where $n_{m}$ is the number of chords of length $m$. These are all measures of domain size that have been used in previous studies. ${ }^{16,20}$

We also define the domain wall densities $\rho_{1}$ and $\rho_{2}$ of type I and type II domain boundaries respectively as the total length (in lattice units) of each domain wall type at saturation divided by the number of fibers in the lattice. Zig-zag domain walls are broken up into vertical and horizontal sections which contribute to the domain wall densities as well.

Figures 6, 7, 8 and 9 summarize the dependence of these measured quantities on $\alpha$ and $\beta$. The effects of the two levels of cooperativity considered are different on the measures of domain size. Consider an already adsorbed particle $M$ at the edge of a growing domain. The $3 \mathrm{NN}$ cooperativity enhances the adsorption of particles that belong to the same domain as $M$. Hence increased $3 \mathrm{NN}$ cooperativity, quantified by a larger value of $\alpha$, corresponds to larger domains. In contrast, the effect of $4 \mathrm{NN}$ cooperativity is to enhance the rate of formation of particles that belong to a different sublattice than that occupied by $M$. This disrupts the growth of the domain by effectively nucleating a new domain on a different sublattice, giving rise to a Type II domain boundary shown in figure 4(c). Hence increasing the level of $4 \mathrm{NN}$ cooperativity, $\beta$, gives rise to smaller domains. This is seen in the measures of average domain size and average chord length from simulation, figures 6 and 7. From this argument, increasing $\beta$ should also increase the density of type II domain walls, as confirmed in figure 9. The density of type I domain walls is essentially the density of unclustered fibers and thus closely related to the saturation coverage (increased $\theta^{*}$ should correspond to a fall

$\uparrow$ The asymptotic size of a single growing domain in an otherwise empty lattice is circular for Arrhenius-type rates. However, at its initial stages of growth the domain is rectangular since rows fill up quickly for Arrhenius rates. If we ignore $4 \mathrm{NN}$ cooperativity for the moment, Evans and Nord ${ }^{21}$ have shown that growing domains retain their rectangular size up to a linear size of order $\alpha^{1 / 2}$ which also turns out to be the typical size a domain grows before it encounters another domain that nucleated independently. Thus domains do not attain their asymptotic shape but remain largely rectangular in the saturated state. As we shall see, the $4 \mathrm{NN}$ cooperativity tends to make domains even smaller and thus preserves their primarily rectangular shapes. See also footnote on page 5 .
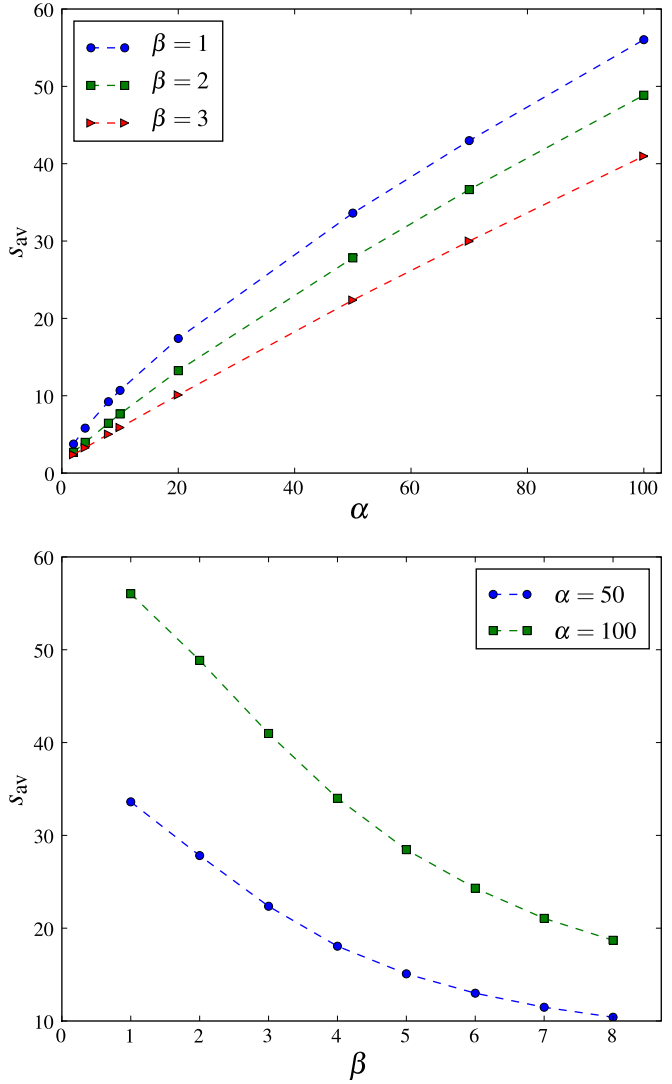

Fig. 6 Dependence of average domain size $s_{\text {av }}$ on cooperativity parameters $\alpha$ and $\beta$ in the CSA model (simulation).

in $\rho_{1}$ as fewer unclustered fibers are left at saturation). Hence increasing $\alpha$ and $\beta$ both have the effect of reducing $\rho_{1}$ (figure 8).

A useful consequence of the competing influence of $3 \mathrm{NN}$ and $4 \mathrm{NN}$ cooperativity on domain size is that the parameters may be varied in tandem to increase the coverage at saturation without simultaneously increasing the average domain size. In previously studied models of cooperative sequential adsorption that include only one level of cooperativity (typically NN enhancement or $\mathrm{NN}$ exclusion and $2 \mathrm{NN}$ enhancement ${ }^{19,20}$ ), increasing the rate of cooperative adsorption increases both the saturation coverage and the size of domains. In contrast, if the system exhibits cooperativity that induces adsorption of particles on a different sublattice as in the current model, then increasing this rate ( $\beta$ in the current model) increases the saturation coverage $\theta^{*}$ while decreasing the measures of domain size, $s_{\mathrm{av}}$ and $m_{\mathrm{av}}$. 

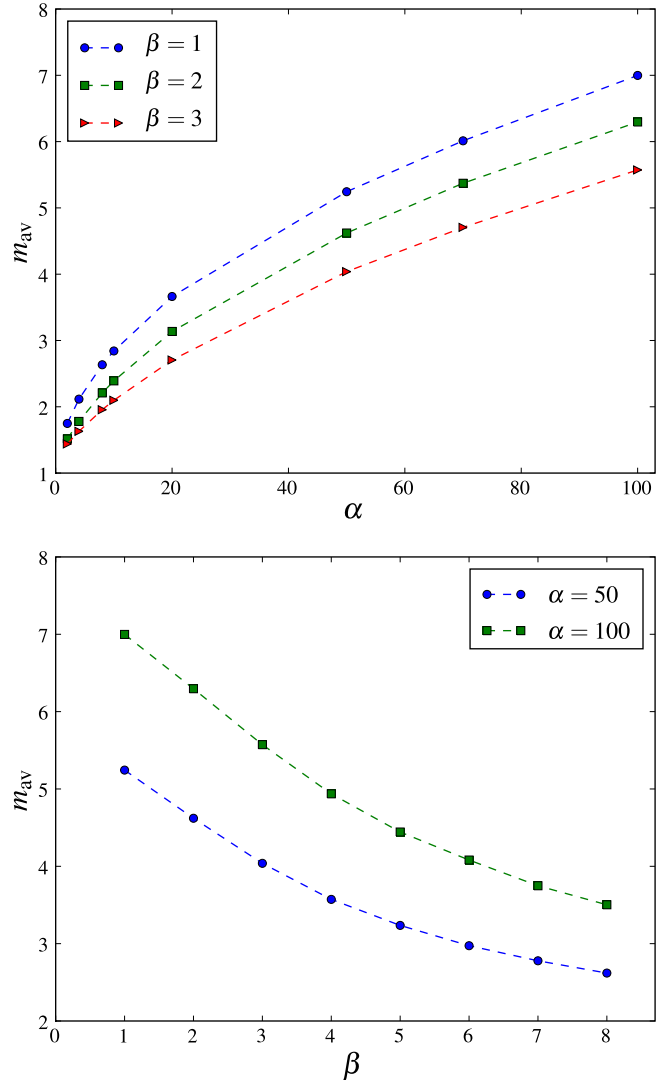

Fig. 7 Dependence of average chord length $m_{\mathrm{av}}$ on cooperativity parameters $\alpha$ and $\beta$ in the CSA model (simulation). Note the qualitatively different trends.

\subsection{Pair correlations}

We define spatial pair correlations at saturation as $C(x, y)=$ $P(x, y)-\theta^{* 2}$, where $P(x, y)$ is the probability that a pair of sites separated by the vector $(x, y)$ in lattice units is occupied. Here we analyze correlations purely in the $x$ direction, $C(x, 0)$, averaged over several simulation runs. The average $x$ and $y$ direction correlations are equal by symmetry. The correlation $C(x, 0)$ is positive for even values of the lattice separation $x$ and negative for odd values as a consequence of nearest-neighbor exclusion in the tetramer model. For instance, $C(1,0)=-\theta^{* 2}$ since $P(1,0)=0$.

\subsubsection{Dependence on 3NN cooperativity}

At first we set $\beta$ to 1 (no $4 \mathrm{NN}$ cooperativity) and vary $\alpha$. The system is then very similar to the $\mathrm{C}(2 \times 2)$ adsorption model with NN exclusion and $2 \mathrm{NN}$ cooperativity with Arrhenius rates studied by Evans and co-workers ${ }^{20,21}$ for which scaling arguments were developed by the authors using semi-
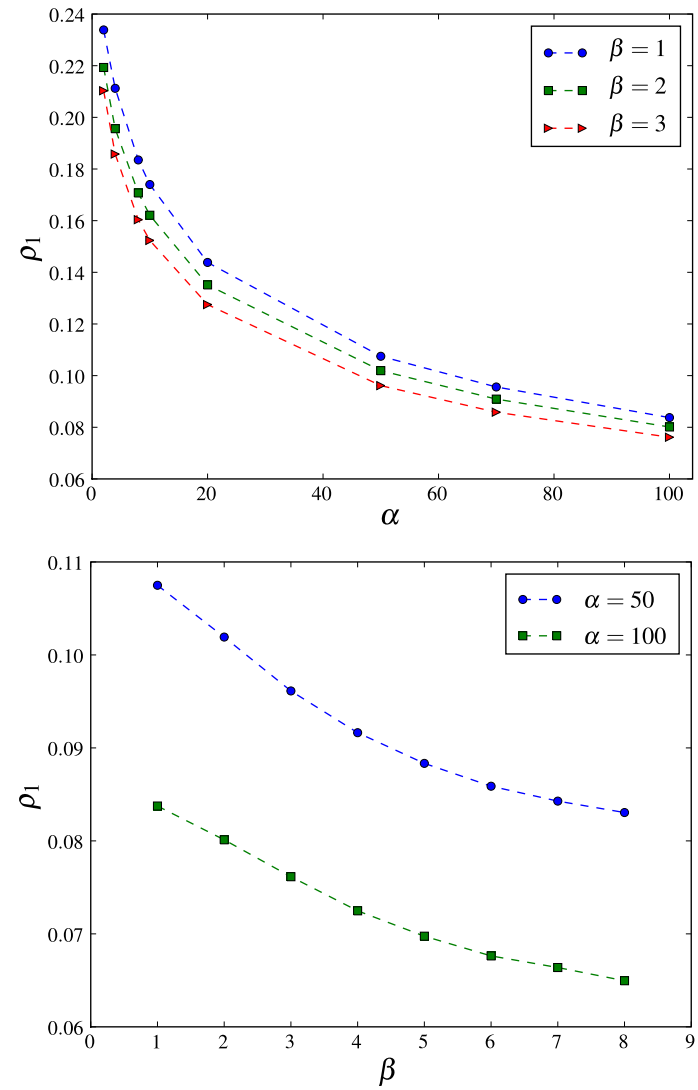

Fig. 8 Dependence of type I domain wall density $\rho_{1}$ on cooperativity parameters $\alpha$ and $\beta$ in the CSA model (simulation).

deterministic domain growth models. For large $\alpha$, the adsorption process resembles a deterministic nucleation and growth process in which new domains are nucleated with a rate $k_{0}$ per empty site, following which rectangular domains grow at a size-dependent rate $\alpha k_{0} m$ where $m$ is the number of adsorbed particles on a growing edge of the domain. (When $\alpha \gg 1$, the time taken for a complete row to be added to the edge of a growing domain, $\sim m /\left(\alpha^{2} k_{0}\right)$, is much smaller than the time taken for the new row to be initiated at that edge which is roughly $1 /\left(m \alpha k_{0}\right)$. The latter is thus the rate-determining step for domain growth.) If fluctuations and edge roughening are ignored, the only relevant quantity is the ratio of the rates of domain nucleation to domain growth. Hence patterns generated by different values of $\alpha$ should be self-similar if they are rescaled by a characteristic length $\xi$ that gives rise to the same nucleation to growth rate ratio for all of them. For Arrhenius rates in the limit of large $\alpha$ in two dimensions, this length has been shown to scale as $\xi \sim O\left(\alpha^{1 / 2}\right)$ (Ref. 21). At high values of $\alpha$, the average linear dimension of the domains and the chord length $m_{\mathrm{av}}$ are expected to scale in the same fashion. 

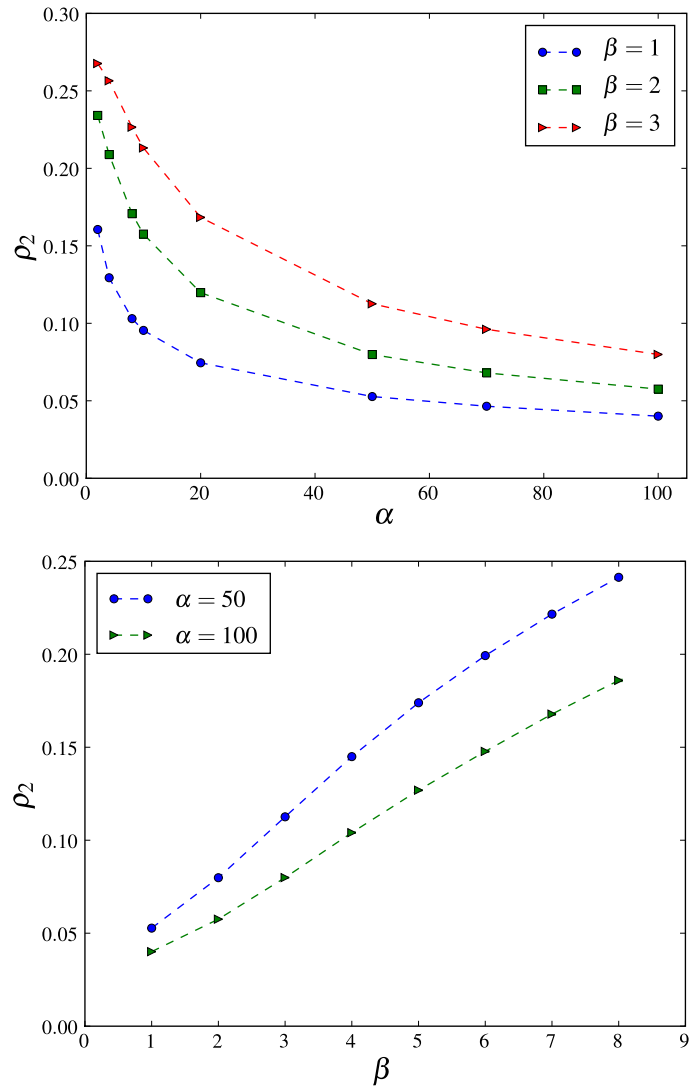

Fig. 9 Dependence of type II domain wall density $\rho_{2}$ on cooperativity parameters $\alpha$ and $\beta$ in the CSA model (simulation).

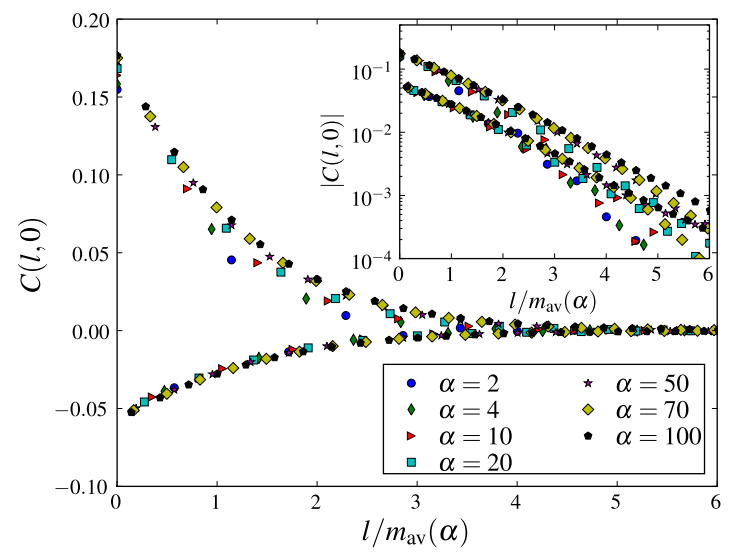

Fig. 10 Pair correlation $C(l, 0)$ for different values of $\alpha$ plotted against the rescaled separation $l / m_{\mathrm{av}}(\alpha)$. In all cases, $\beta=1 . m_{\mathrm{av}}(\alpha)$ is taken from data similar to that reported in figure 7 with $\beta=1$.For $\alpha>20$ the scaled correlations follow a universal form. The inset, which plots the absolute value of the pair correlation on a log-linear scale, shows the loss of scaling at large separations.

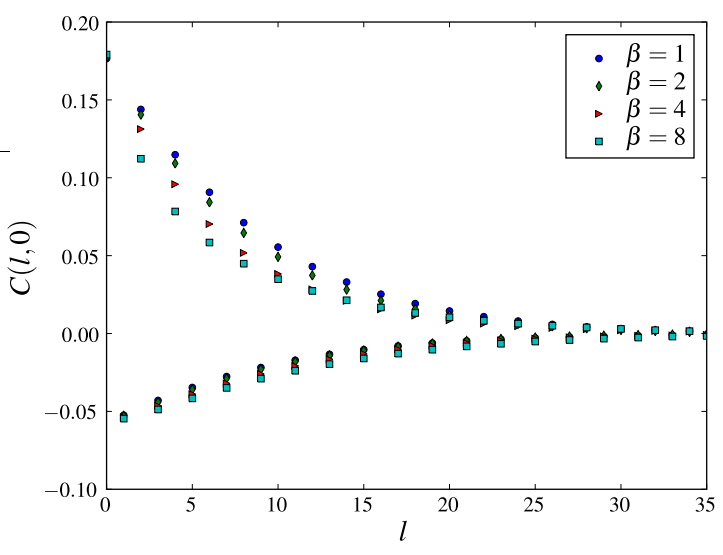

Fig. 11 Pair correlation $C(l, 0)$ for $\alpha=100$ and different values of $\beta$ against separation $l$ in lattice units.

A consequence of the length scale set by the strong cooperativity is that the spatial correlations should display universal scaling on length scales $O(\xi)$ for large values of $\alpha$. On length scales larger than the characteristic length, a crossover to the superexponential decay characteristic of random sequential adsorption processes is expected. ${ }^{20,25}$ Such behavior is indeed seen in figure 10 .

\subsubsection{Dependence on $4 \mathrm{NN}$ cooperativity}

The effect of varying $\beta$ while keeping the value of $\alpha$ constant is shown in figure 11. As $\beta$ is increased, the positive-valued part of the correlation function $(C(l=2 n, 0)$ where $n$ is an integer) falls. This is a result of the increased propensity for type II domain walls to form, which break up contiguous strings of adsorbed particles on the same sublattice in the $x$ direction (see figure 4(c)). On the other hand, the negative-valued part $(C(x=2 n+1,0))$ becomes more negative, because a particle in the domain on the left of figure 4(c) continues to have vacancies at positions that are odd numbers of lattice displacements along the same row into the new domain on the right.

The pair correlations in figure 11 cannot be made to overlap by rescaling the horizontal axis. (For instance, if we rescaled the $l$ axis to make the positive-valued parts of $C(l, 0)$ overlap for $\beta=1$ and $\beta=8$, we can see from the figure that the negative-valued parts of the curves would be pushed even further away compared to the unscaled functions.) The simple scaling of correlations seen when $\beta$ was set to 1 has been lost. In the former case, scaling was justified by considering a deterministic domain nucleation and growth process where the ratio of the rates of domain nucleation to growth set the the characteristic length scale. When $4 \mathrm{NN}$ cooperativity is also included, the deterministic process is modified as follows: nucleation still happens at a rate $k_{0}$ per empty lattice site, and 


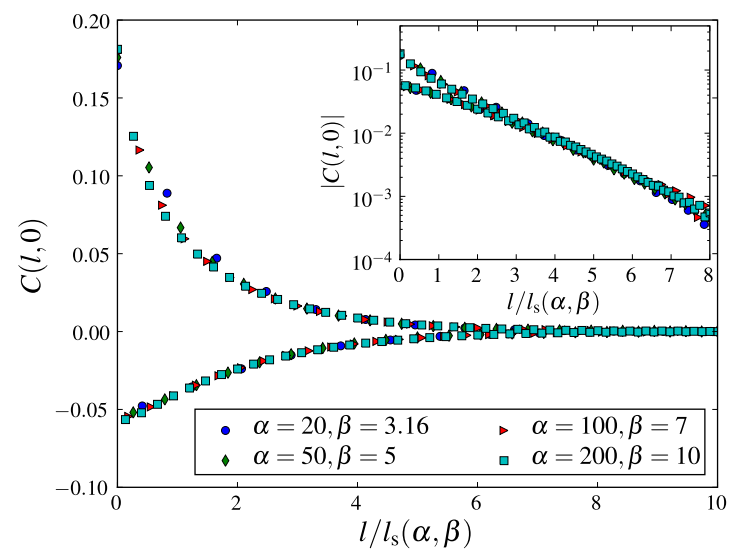

Fig. 12 Pair correlation $C(l, 0)$ for different values of $(\alpha, \beta)$ chosen such that $\alpha / \beta^{2}=2$ in all cases. The scaling length $l_{s}(\alpha, \beta)$ was chosen for each value of $(\alpha, \beta)$ to obtain the best data collapse. Inset: $|C(l, 0)|$ against $l / l_{s}$ on a $\log$-linear scale.

rows continue to be added to the straight edge of a growing domain at a rate $m \alpha k_{0}$ where $m$ is the number of particles at the edge. However, domain growth (where a row is added to the same sublattice as the domain) now competes with the formation of a new domain on the sublattice displaced by one lattice step in either direction perpendicular to the growing edge, which happens at a rate $\sim m \beta^{2} k_{0}$. The deterministic process thus depends on two rate ratios: the ratio of domain nucleation to growth, which is domain size dependent but controlled by $\alpha$, and the ratio of domain growth to formation of a new domain at the growing edge, which equals $\alpha / \beta^{2}$ for all sizes of domains.

The pair correlations do not scale as before because it is not possible to find a single characteristic length for rescaling that sets both rate ratios to be equal for patterns with different values of $\alpha$ and $\beta$. However, we expect that patterns with the same value of the ratio $\alpha / \beta^{2}$ may be made self-similar by the appropriate rescaling that sets the nucleation-growth ratio to be uniform. This is confirmed in figure 12 where pair correlations for different values of $(\alpha, \beta)$ but the same value of $\alpha / \beta^{2}$ have the same form upon appropriate rescaling of the horizontal axis. Note that for each of the values of $\alpha$, a different value of $\beta$ would not give a pair correlation that could be collapsed onto the same curves. As before, the conclusions drawn from the deterministic model are appropriate only for large values of $\alpha$ where the domain nucleation and growth picture is not completely obscured by fluctuations due to the stochastic nature of the sequential adsorption model.

From the above discussion, we also see that the ratio $\alpha / \beta^{2}$ quantifies of the relative influence of the $3 \mathrm{NN}$ and $4 \mathrm{NN}$ cooperativity on domain sizes. If $\alpha / \beta^{2} \ll 1$, the $4 \mathrm{NN}$ cooperativity dominates, disrupting domain growth completely even for large $\alpha$ because it would be much more likely for a new row of tetramers to form on a shifted sublattice than on the same sublattice as a previously formed row. In that situation one would not observe large ordered domains at all, but rather see regions of staggered parallel rows of tetramers on alternating sublattices. The case of $\alpha / \beta^{2} \ll 1$ is not relevant to the microfiber clustering system (for which domains extending over a few lattice lengths in either direction are always observed, indicating that $3 \mathrm{NN}$ cooperativity is dominant) and is not considered here.

\subsection{Diffracted intensity}

Although one does not typically diffract matter or light waves from the tetramer patterns studied here, Fourier analysis nevertheless provides a powerful tool for uncovering subtle patterns underlying tetramer formation. We define the diffracted intensity of the dual lattice of tetramers at a wavevector $\mathbf{q}$ as

$$
I(\mathbf{q})=\left|\sum_{x, y} e^{-i\left(q_{x} x+q_{y} y\right)} n(x, y)\right|^{2}
$$

where $q_{x}, q_{y} \in(-\pi, \pi), n(x, y)$ is the occupation number ( 0 or 1 ) of the lattice position $(x, y)$ and the sum runs over all lattice positions. This quantity is closely related to the diffracted intensity for scattering from the actual pattern of tetramers; it is the pattern that would be obtained if every tetramer in the pattern were replaced by a delta function scatterer at its center (similar to the structure factor for scattering from a collection of identical atoms). It provides information about the degree of order in the system and the types of domains and domain boundaries that are present.

In practice, the results of simulations and the experimental measurements are discrete arrays of size $N \mathrm{x} N$. In this case we calculate the diffracted intensity via a discrete Fourier transform of the array $n(x, y)$ of occupation numbers. The resulting array provides an estimate of $I\left(q_{x}, q_{y}\right)$ for $q_{x}, q_{y}$ taking on discrete values $2 \pi n / N$ where $n$ takes on integer values between $-N / 2$ and $N / 2$. Averaging the calculated patterns from several realizations of a finite-sized simulation of tetramer deposition at a particular $(\alpha, \beta)$ provides a diffraction pattern representative of that set of parameters.

A single domain is a square array of particles with a superlattice spacing of twice the underlying lattice. The diffraction pattern of an infinite domain consists of delta function peaks at $\mathbf{q}= \pm(\pi, 0), \pm(0, \pi), \pm(\pi, \pi), \pm(\pi,-\pi)$, etc., i.e. the reciprocal lattice of the square superlattice of particles, in addition to the origin. At finite cooperativity, finite-sized domains on different sublattices interfere to broaden these peaks in specific ways. To understand this, suppose we start with an infinite domain and create a Type II domain wall running along the $y$ direction by shifting all the particles to the right of the origin up 

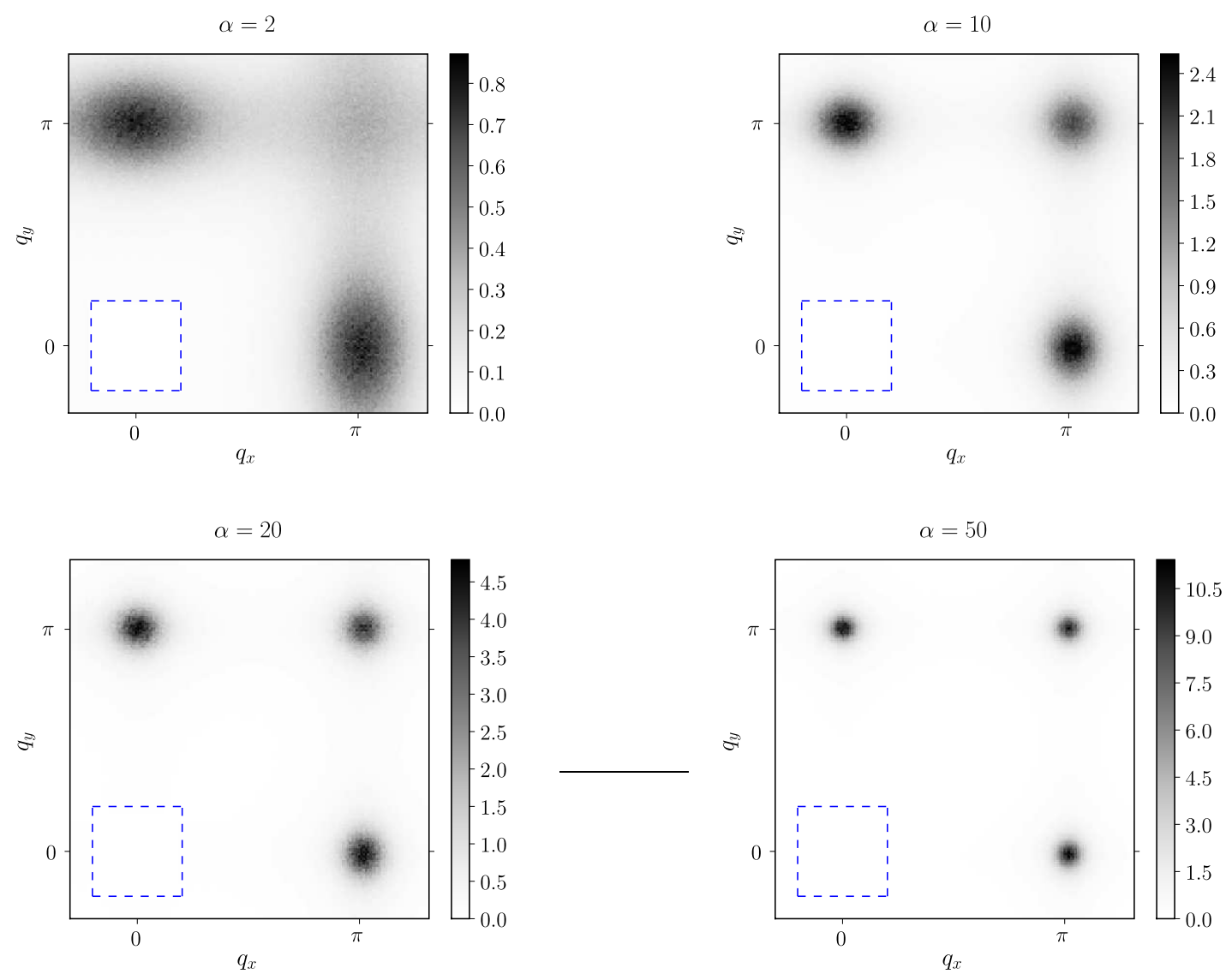

Fig. 13 Intensity plots of diffraction patterns from simulations for different values of $\alpha$. Since the pattern is fourfold symmetric, only one quadrant is shown but the $x$ and $y$ axes have been offset to reveal the shapes of the diffraction spots. The square region $-\pi / 5<q_{x}, q_{y}<\pi / 5$ (dotted line) has been set to zero to emphasize the features near the edges. There is no $4 \mathrm{NN}$ cooperativity; i.e. $\beta=1$ in all cases.

by one lattice position. The $x$ coordinates of all occupied sites remain the same in the sum in equation 1 ; hence the peaks at $\mathbf{q}=( \pm \pi, 0)$ are unaffected but the peaks at nonzero values of $q_{y}$ broaden in the $q_{x}$ direction. ${ }^{\star}$ Analogously, Type II domain walls in the $x$ direction broaden the peaks with $q_{x} \neq 0$ in the $q_{y}$ direction. Type I domain walls have an effect of either diminishing the strength of peaks or broadening them perpendicular to their non-zero momentum direction.

Increasing $\alpha$ while setting $\beta=1$ constant (figure 13) sharpens the principal reciprocal lattice peaks by reducing domain wall densities, allowing large domains to dominate the diffraction pattern. At a constant value of $\alpha$, increasing the value of $\beta$ (figure 14) increases Type II domain wall densities exclusively, thus broadening the peaks at $(0, \pm \pi)$ and $( \pm \pi, 0)$

$\ddagger$ The width of a diffraction peak in either direction is inversely proportional to the correlation length of the pair correlation in that direction. The domain wall reduces pair correlations in the $x$ direction (by dephasing rows of adsorbed particles that extended across the entire lattice) but not in the $y$ direction, hence affecting the peak width along $q_{x}$ but not $q_{y}$. perpendicular to the non-zero momentum and diminishing the peaks at $( \pm \pi, \pm \pi)$.

\section{Experimental results}

To test our theoretical ideas, we conducted clustering experiments on a sample of size $2 \mathrm{~cm} \times 1 \mathrm{~cm}$, with fibers of height $10 \mu \mathrm{m}$ and diameter $1.8 \mu \mathrm{m}$ arranged in a square array with lattice constant $3.5 \mu \mathrm{m}$ (Sample I). Experimental details are in Appendix B. Optical microscope images were taken of a 182 x 182 fiber area at a time, and a pattern recognition program written in MATLAB ${ }^{\circledR}$ (MathWorks, Inc.) was used to identify the positions of the formed tetramers on the dual lattice. Thus a 182 x 182 lattice of empty and filled sites was obtained from each image, the filled sites being the ones at which a tetramer was observed. From these lattices, relevant measures such as the coverage, domain sizes and chord lengths could be measured which correspond exactly to the quantities mea- 

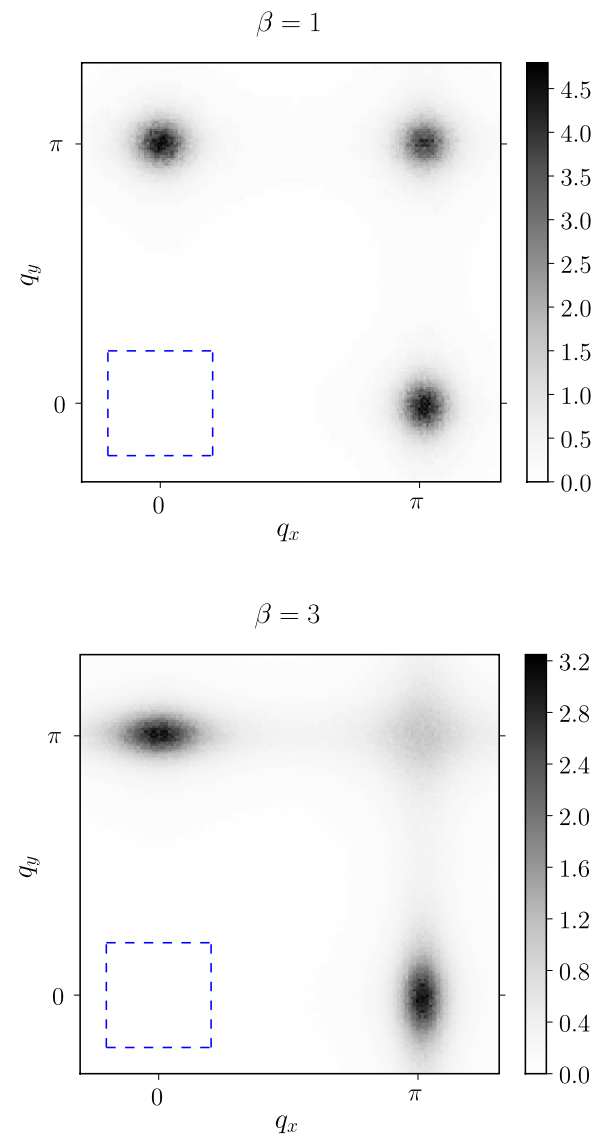
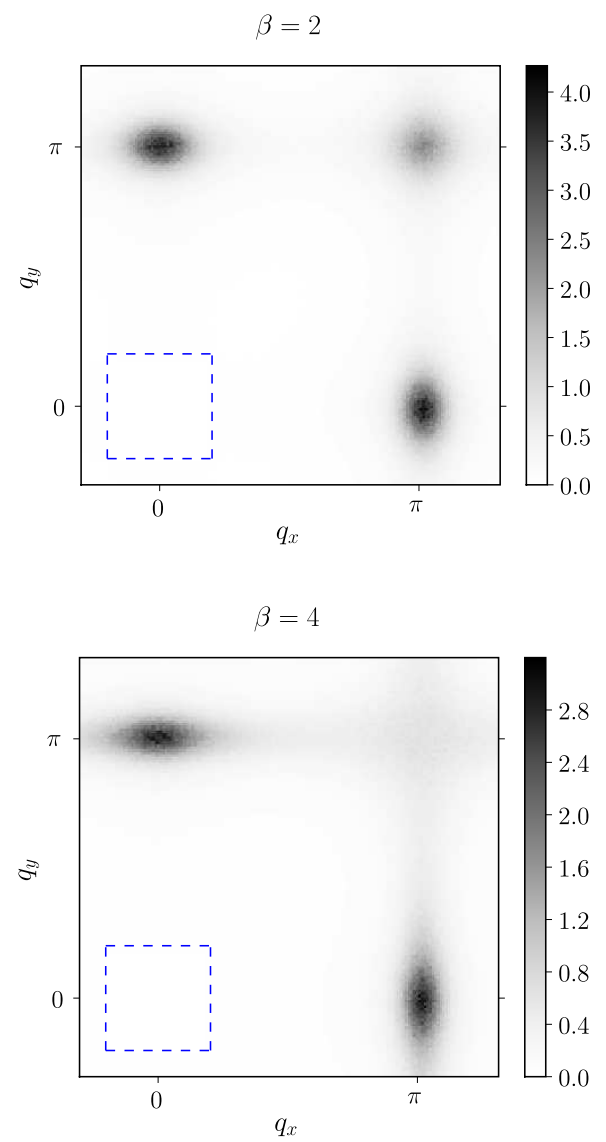

Fig. 14 Intensity plots, similar to figure 13, of diffraction patterns from simulations for different values of $\beta$. In all cases, $\alpha=20$.
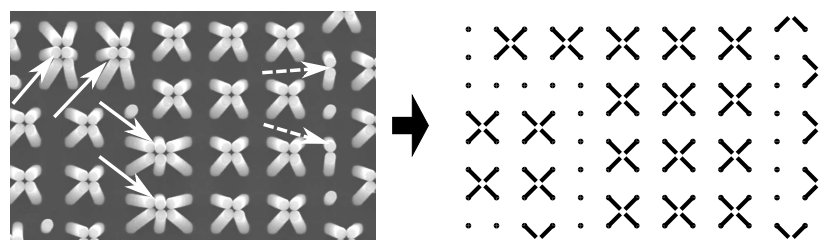

Fig. 15 A section of an experimental image (left) compared to its interpretation in terms of tetramers on a lattice (right) within the framework of our model. Notice especially that the hexamer $(3 \times 2)$ clusters, indicated by solid arrows, have been interpreted as being parts of a continuous type I domain boundary. Similarly, dimers that form along another type I boundary, indicated by dashed arrows, are interpreted as a row of lone fibers.

sured from the CSA model simulations. We imaged 43 nonoverlapping regions, and calculated pattern statistics for each lattice. Here we report quantitites averaged across the 43 re- gions, and the corresponding standard error of this average is used as the error estimate. ${ }^{\S}$

In the experiment, we also see clusters that do not strictly consist of four fibers, such as hexamers ( $3 \times 2$ clusters). These are primarily seen at the boundaries between ordered domains belonging to different sublattices. We interpret these clusters in the context of the tetramer adsorption model as follows: the ethanol-air interface is pinned to the tips of the fibers even as the level of the ethanol-air interface recedes below the height of the fibers. The capillary forces between fiber tips are a result of this pinning which perturbs the interface, and they

$\S$ The standard error, $s=\sigma / \sqrt{n}$ where $\sigma$ is the estimate of the standard deviation of the measured quantity and $n$ is the number of measurements taken, is a valid estimate of the error associated with measuring a mean quantity, as long as the individual measurements are made from the same statistical distribution with a uniform value of $\sigma$. In practice, slight nonuniformities in the sample, as well as spatial irregularities in the evaporation rate of ethanol, are likely to make the cooperativity levels in each of the 43 regions slightly different from one another; i.e. the measurements made in each region belong to slightly different statistical distributions. This variation is not reflected in the standard error which is thus likely to underestimate the true errors in the measured quantities. 
increase as the height difference between fiber tips and interface increases. ${ }^{23}$ These capillary forces compete with the elastic forces involved with bending the fibers so that their tips touch to form clusters. When the level of the ethanolair interface is such that capillary forces are strong enough to induce tetramer clustering, tetramer formation begins and proceeds until saturation; larger cluster formation does not happen because fiber tips need to be displaced by larger distances, and higher capillary forces are needed to overcome the corresponding elastic forces. If the evaporation were stopped at this stage a pattern consisting only of tetramers and upright (unclustered) fibers would be observed. However, in the actual experiments, ethanol evaporation continues until the capillary forces become strong enough to draw some of the remaining lone fibers toward their neighbors to form dimers (clusters of two adjacent pillars), hexamers and larger sized clusters. Thus we interpret these clusters as a combination of tetramers and lone fibers when we convert the experimental images into lattice occupancy data. To be consistent with this interpretation, we translate a row of hexamers in the experiment as a row of tetramers next to a row of unclustered fibers (rather than randomly assigning a tetramer to one of the two positions it could occupy in each hexamer) because such a row is most likely to have arisen at the site of a continuous Type I domain wall which we recreate in the lattice data. Figure 15 shows an example of translating an experimental pattern into lattice data.

The method of fitting the experimental results to the simulations is as follows: the two-dimensional diffracted intensity defined in equation 1 condenses ensemble-wide properties of the generated patterns into a two-dimensional array of numbers. We thus compare the averaged diffracted intensity from the experiment to that from the simulations, choosing $\alpha$ and $\beta$ that best fit the experimental diffraction pattern. The parameters are chosen that minimize the root mean square deviation of the diffracted intensity, weighted by the inverse of the experimental error estimate at each $\left(q_{x}, q_{y}\right)$. As figure 16 shows, such a minimum value may be extracted from the data to a reasonable precision. For our experimental measurements, we obtain $\alpha=48 \pm 1, \beta=2.7 \pm 0.1$.

Table 1 shows a comparison between experiment (second column) and the CSA model simulation for the optimized $\alpha$ and $\beta$ values described above (third column). The measured average values of the different pattern statistics introduced previously appear to be in reasonable agreement. Figure 17 compares the calculated pair correlation function from the experiment to that of the CSA model with the chosen fit parameters, again showing reasonable agreement.

We can also compare the performance of the extended CSA model to a one-parameter model with only $3 \mathrm{NN}$ cooperativity and Arrhenius rates $k_{i}=k_{0} \alpha^{i}$ for an empty site with $i$ occupied sites at third nearest neighbor positions (this is equivalent
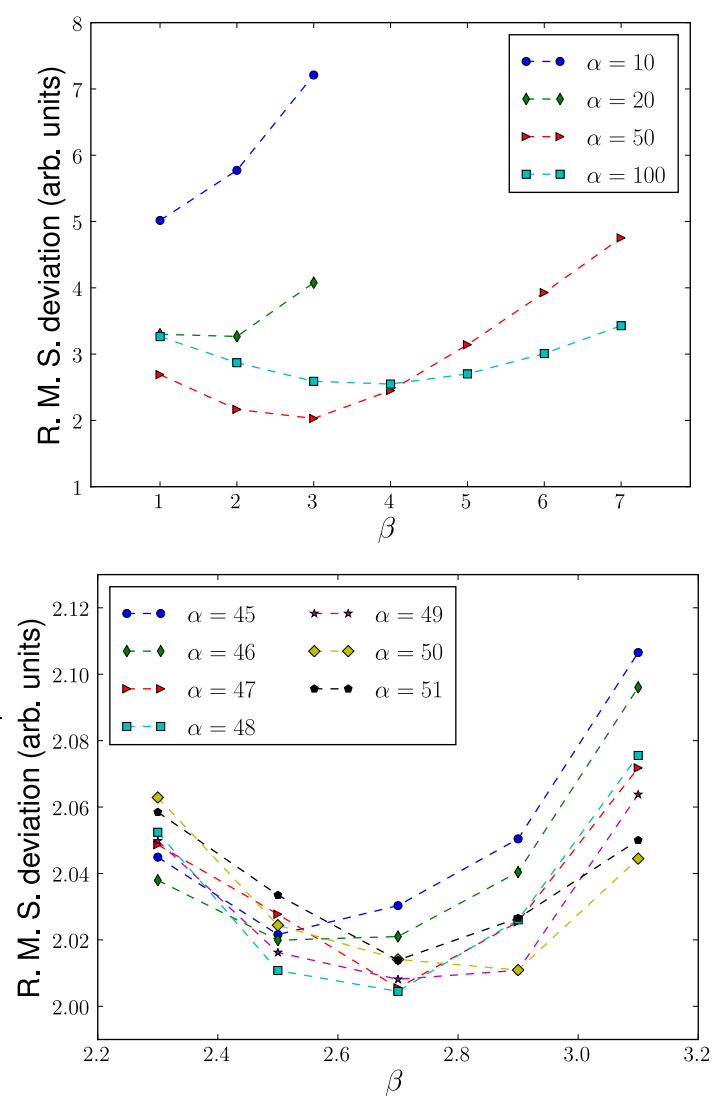

Fig. 16 Least-squares fitting of the experimental result to simulations. Each line shows the RMS value of the difference in observed and simulated diffraction intensity (averaged across the twodimensional array) for a particular value of $\alpha$ while varying $\beta$. On the top, the smallest value of of the RMS deviation decreases and then increases upon varying $\alpha$ over a large range, suggesting that a unique minimum can be found in the vicinity of $\alpha=50, \beta=3$. On the bottom, more refined parameters $\alpha=48, \beta=2.7$ are seen to provide the best fit with a precision of \pm 1 in $\alpha$ and \pm 0.1 in $\beta$.

to the two-parameter model with $\beta$ set to 1). Statistics for the best fit obtained for $\alpha$ in the one-parameter model have also been reported in table 1 (fourth column), showing that the two parameter model performs significantly better. In particular, the absence of $4 \mathrm{NN}$ cooperativity leads the one-parameter model to greatly underestimate $\rho_{2}$, the density of type II domain boundaries which are induced by the $4 \mathrm{NN}$ cooperativity mechanism. The high value of $\rho_{2}$ in the experiment confirms that the $4 \mathrm{NN}$ cooperativity mechanism is important in tetramer formation. 


\begin{tabular}{cccc}
\hline Quantity & Experiment & $\begin{array}{c}\text { Two-parameter CSA } \\
\alpha=48, \beta=2.7\end{array}$ & $\begin{array}{c}\text { One-parameter CSA } \\
\alpha=41\end{array}$ \\
\hline$\theta^{*}$ & $0.2258 \pm 0.0004$ & 0.2253 & 0.2213 \\
$s_{\mathrm{av}}$ & $20.6 \pm 0.5$ & 23.2 & 29.1 \\
$m_{\mathrm{av}}$ & $4.0 \pm 0.1$ & 4.12 & 4.84 \\
$\rho_{1}$ & $0.099 \pm 0.002$ & 0.099 & 0.115 \\
$\rho_{2}$ & $0.111 \pm 0.002$ & 0.105 & 0.057 \\
\hline
\end{tabular}

Table 1 Comparison of measured quantities from Sample I with averaged statistics of the two-parameter and the one-parameter CSA models with parameters picked by least-squares fitting of the diffraction pattern with experiment. The simulation results are averaged over 100 instances of a $400 \times 400$ lattice and the standard error associated with this average is smaller than the most significant digit reported.

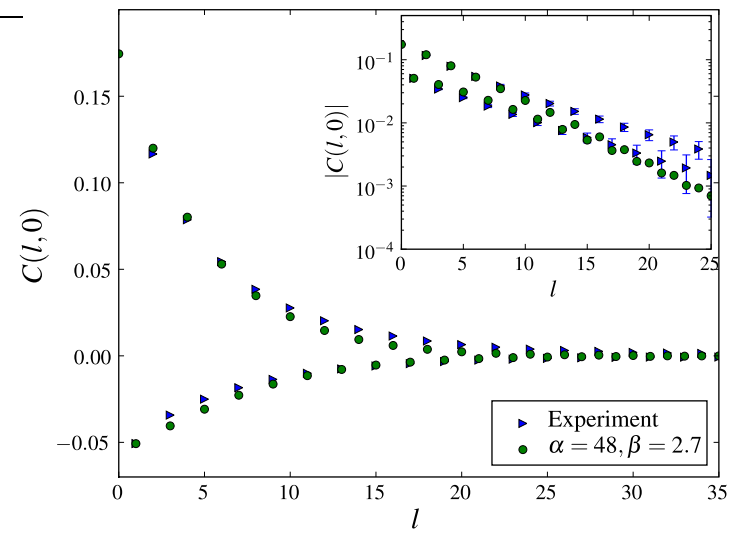

Fig. 17 Comparison between the measured pair correlation of Sample I to the pair correlation function of the CSA model with $\alpha=$ $48, \beta=2.7$. Inset: Absolute value of the pair correlations on a $\log$ linear scale.

\section{Conclusion}

We have proposed a lattice sequential adsorption model of ordered tetramer clusters of microfibers driven by capillary forces. The newly introduced further-neighbor cooperativity allows independent variation of lattice coverage and average domain size: unlike previously studied cooperative sequential adsorption models, the present model allows the saturation lattice coverage to be increased without correspondingly raising the average domain size. This independence arises because the model admits additional domain boundaries that do not include unoccupied sites.

With a plausible model for ordered cluster formation as judged by the comparison of the theory to experiment, we may ask how to improve ordering in the capillary-assisted self-organization process. Clearly, increasing the rate of cooperative domain growth relative to random domain nucleation gives rise to larger ordered domains. A low level of the ethanol-air interface relative to the height of the fiber tips increases the downward as well as inter-fiber-tip capillary forces, which would induce more random nucleation events. If we control the evaporation of the ethanol (for instance, by controlling the ambient vapor pressure of ethanol) in such a way that the interface spends a longer time at a height that induces few tetramers to form on their own, then clustering is more likely to be triggered among fibers destabilized by the formation of clusters nearby than by the effect of the capillary forces on upright fibers. Thus the rate of bare nucleation of domains is reduced relative to their growth rate, and larger ordered domains could be produced.

The $4 \mathrm{NN}$ cooperativity is also a factor that leads to smaller domains. Indeed, even if domain growth arises from a single nucleation site and further isolated single-site nucleation is suppressed, there is always a chance that a domain belonging to a different sublattice begins somewhere along the growing edges of the domain as long as the $4 \mathrm{NN}$ cooperativity is significant. A second alternative to create uniform domains is to create artificial domain nucleation sites (say, by weakening or bending individual fibers as has been demonstrated in Ref. 22) in a regular square array with each nucleation site created on the same sublattice and the spacing between nucleation sites smaller than the typical domain size observed in an uncontrolled clustering experiment. This strategy would enhance domains on the same sublattice and allow commensurate coalescence before random single-site nucleation or $4 \mathrm{NN}$ cooperativity-induced nucleation could initialize a domain belonging to a different sublattice.

A similar effect can be recreated along one dimension by imposing clustering dynamics at a front that sweeps across the sample in one direction rather than allowing random nucleation and bidirectional domain growth everywhere in the sample, as has been previously recognized in Ref. 13. This bias can be achieved, for instance, by performing the clustering on a tilted sample. Due to the effect of gravity, the wetting layer retreats in one direction as the ethanol evaporates, and at any moment clustering happens only in a few horizontal rows near the retreating edge where the wetting layer is at its thinnest. Domain growth along the horizontal strip happens very quickly under $3 \mathrm{NN}$ cooperativity, following which the 

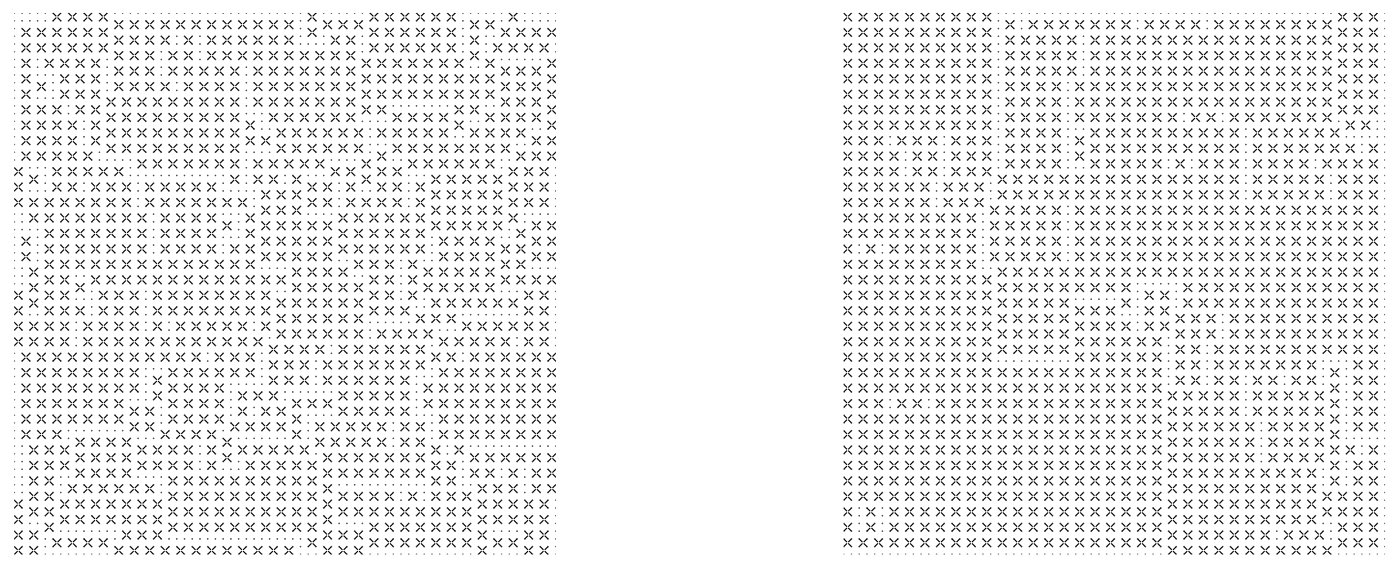

Fig. 18 Single simulation result for (left) CSA with $\alpha=40, \beta=2$ (left) compared with (right) a simulation with the same parameters in which clustering happens only in a strip three rows wide that is swept across the lattice from top to bottom to mimic the effect of an evaporation front (see text). There are far fewer domain boundaries in the row-by-row simulation on the right, and they tend to lie along the vertical axis.

domain edge acts as a template for clustering in the next unclustered row. This leads to extended domains in the direction of propagation of the clustering front (top to bottom), while in the perpendicular (horizontal) direction domains are broken up by $4 \mathrm{NN}$ cooperativity and random nucleation. A pattern resulting from a simulation of such a mechanism is shown in figure 18 .

Finally we remark that this work could be relevant to future studies of various self-organization processes as well as sequential adsorption. For example, this model could be used to describe nonequilibrium adsorption of a molecule/complex/particle/DNA tile that has a square/cross symmetry with the neighboring corners/ligands/arms possessing either opposite charge, or A,B,A,B recognition sites. Then the attachment of the next particle is mostly enhanced at the NN position where a bidentate junction is formed, but it will be also enhanced (but to a lesser degree) at the $2 \mathrm{NN}$ position where a monodentate junction is formed. Again, we arrive at double-level cooperativity in adsorption. The difference is that there will be no NN/2NN exclusion, but instead $\mathrm{NN} / 2 \mathrm{NN}$ enhancement; otherwise, the model is largely the same. Abstraction of complex processes to lattice-type models that do not focus on microscopic details has been used with success to describe self-assembly of nanoparticles ${ }^{26}$ and vesicle formation ${ }^{27}$ among other processes; here we use an out-of-equilibrium lattice process to study a microscale selforganization process. The two-parameter sequential adsorption model we have developed could be relevant to more 'traditional' sequential adsorption processes of gas molecules on metal surfaces where further neighbor interactions are significant, and also to other irreversible processes that are not adsorption processes in the strict sense but nevertheless may be illuminated using such models.

\section{A Details of numerical simulation}

The CSA process was simulated in a program written in the $\mathrm{C}++$ programming language. For each realization of the adsorption process for a particular set of $(\alpha, \beta)$ values, a twodimensional binary state array ( $0 \equiv$ "empty"; 1 三 "occupied") of size equal to that of the lattice being simulated was initialized to zero (all positions vacant). Periodic boundary conditions were used to minimize finite-size effects. Initially all sites were assigned the same rate $k_{0}=1$ in arbitrary units. At each iteration of the sequential process, a site was chosen at random for an adsorption trial. The probability of a site being chosen was set to be proportional to the rate of adsorption assigned to it. If adsorption of a particle was allowed by the exclusion rules, the state of that site was changed to "occupied" and the adsorption rate of vacant sites at $3 \mathrm{NN}$ and $4 \mathrm{NN}$ positions was updated using the set values of $\alpha$ and $\beta$. The iterations continued until no further adsorption was allowed by the exclusion rules. Since the evolution in time of the process was not of interest, the simulation was sped up by periodically eliminating sites at which adsorption was excluded from consideration as adsorption candidates so that the frequency of unsuccessful adsorption attempts remained low and the jammed state was attained quickly.

\section{B Experimental}

The microfiber array was prepared in epoxy, using a polydimethylsiloxane (PDMS) mold fabricated from a silicon master. The fabrication of the mold is described in detail in Ref. 22. The epoxy used was UVO-114 single component UVinitiated epoxy (Epoxy Technology, Inc.). Epoxy was poured into the PDMS mold using a pipette and allowed to cure un- 
der a B-100 UV lamp (UVP Blak-Ray) for 20 minutes, after which the mold was peeled off. The hardened epoxy sample was exposed to plasma in a FEMTO plasma system (Diener Electronic) for 20 seconds to improve its wettability. To induce clustering, the sample was placed on a flat surface and $10 \mu \mathrm{l}$ of absolute anhydrous ethanol was dropped on it using a pipette. The ethanol was allowed to evaporate under ambient conditions.

Optical microscope mages of the clustered microfiber sample used for pattern recognition were taken using a Leica DMRX microscope connected to a QImaging Evolution VF CCD camera. SEM images used for illustrations in the main text were recorded with a JEOL JSM-6390 scanning electron microscope.

\section{Acknowledgements}

J. P. thanks B. Pokroy and S. H. Kang for guidance in conducting the experiments, F. Gibaud for use of his pattern recognition program and all three for insightful discussions. Work by J. P. and D. R. N. supported by the National Science Foundation through a Harvard University Materials Research Science and Engineering Center Grant DMR-0820484 and through Grant DMR-0654191. Work by J. A. supported through MRSEC grant DMR-0820484 as well.

\section{References}

1 Y. Xia, B. Gates and Z. Y. Li, Adv. Mater., 2001, 13, 409413.

2 P. Rothemund, Proc. Nat. Acad. Sci. USA, 2000, 97, 984.

3 A. Cohen and L. Mahadevan, Proc. Nat. Acad. Sci. USA, 2003, 100, 12141.

4 J. Bico, B. Roman, L. Moulin and A. Boudaoud, Nature, 2004, 432, 690.

5 C. Py, R. Bastien, J. Bico, B. Roman and A. Boudaoud, Europhys. Lett., 2007, 77, 44005 (5pp).

6 Y. Zhao and J. Fan, Appl. Phys. Lett., 2006, 88, 103123.

7 J. Fan, D. Dyer, G. Zhang and Y. Zhao, Nano Lett., 2004, 4, 2133-2138.

8 J. Fan and Y. Zhao, Langmuir, 2006, 22, 3662-3671.

9 D. Chandra, S. Yang, A. A. Soshinsky and R. J. Gambogi, ACS Appl. Mater. Interfaces, 2009, 1, 1698-1704.

10 H. Segawa, S. Yamaguchi, Y. Yamazaki, T. Yano, S. Shibata and H. Misawa, Appl. Phys. A: Mater. Sci. Process., 2006, 83, 447-451.

11 D. Chandra, J. Taylor and S. Yang, Soft Matter, 2008, 4, 979-984.
12 B. Pokroy, S. H. Kang, L. Mahadevan and J. Aizenberg, Science, 2009, 323, 237-240.

13 D. Wu, B. Xu, J. Jiao, Y. Xu, H. Xia and H. Sun, Appl. Phys. Lett., 2009, 95, 091902.

14 A. Sidorenko, T. Krupenkin, A. Taylor, P. Fratzl and J. Aizenberg, Science, 2007, 315, 487.

15 D. Chandra and S. Yang, Langmuir, 2009, 25, 1043010434.

16 J. W. Evans, Rev. Mod. Phys., 1993, 65, 1281.

17 F. Wu, Int. J. Mod. Phys. B, 2006, 20, 5357-5371.

18 D. E. Sanders and J. W. Evans, Phys. Rev. A, 1988, 38, 4186.

19 J. W. Evans, J. A. Bartz and D. E. Sanders, Phys. Rev. A, 1986, 34, 1434.

20 J. Evans, R. Nord and J. Rabaey, Phys. Rev. B, 1988, 37, $8598-8611$.

21 J. Evans and R. Nord, J. Vac. Sci. Technol., A , 1987, 5, 1040.

22 B. Pokroy, A. Epstein, M. Persson-Gulda and J. Aizenberg, Adv. Mater., 2009, 21, 463-469.

23 P. A. Kralchevsky and K. Nagayama, Adv. Colloid Interface Sci., 2000, 85, 145-192.

24 F. Gibaud and J. Aizenberg, high-speed video of cluster formation, unpublished.

25 J. W. Evans, D. R. Burgess and D. K. Hoffman, J. Math. Phys., 1984, 25, 3051-3063.

26 E. Rabani, D. R. Reichman, P. L. Geissler and L. E. Brus, Nature, 2003, 426, 271-274.

27 A. Bernardes, Langmuir, 1996, 12, 5763-5767. 